\title{
Aligned Graphene Mesh Supported Double Network Natural Hydrogel Conduit Loaded with Netrin-1 for Peripheral Nerve Regeneration
}

Qun Huang, ${ }^{\mathrm{a}, 1}$, Yuting Cai ${ }^{\mathrm{b}, \mathrm{d}, 1}$, Xing Zhang ${ }^{\mathrm{a} 1}$ MD, Junchao Liu ${ }^{\mathrm{a}}$, Zhenjing Liu ${ }^{\mathrm{d}}$, Bo Li ${ }^{\mathrm{a}}$, Hoilun Wong ${ }^{\mathrm{d}}$, Feng Xu $\mathrm{X}^{\mathrm{e}}$, Liyuan Sheng ${ }^{\mathrm{f}}$, Dazhi Sun ${ }^{\mathrm{b} *}$, Jinbao Qin ${ }^{\mathrm{ac}}$, Zhengtang Luo ${ }^{\mathbf{d}^{*}}$, Xinwu $\mathrm{Lu}^{\mathrm{ac} *}$

a Department of Vascular Surgery, Shanghai Ninth People's Hospital, Shanghai JiaoTong University, School of Medicine, Shanghai 200011, China

b Department of Materials Science and Engineering, Southern University of Science and Technology, Shenzhen, Guangdong 518055, China

c Vascular Center of Shanghai JiaoTong University, Shanghai, 200011, China

d Department of Chemical and Biological Engineering, and William Mong Institute of Nano Science and Technology, the Hong Kong University of Science and Technology, Clear Water Bay, Kowloon, Hong Kong, China

e Bioinspired Engineering and Biomechanics Center, Xi'an Jiaotong University, Xi'an, Shaanxi, China

${ }^{\mathrm{f}}$ Shenzhen Institute, Peking University, Shenzhen 518057, China

${ }^{*}$ To whom correspondence should be addressed. E-mail: luxinwu@shsmu.edu.cn (X. Lu), keztluo@ust.hk (Z. Luo), sundz@sustech.edu.cn (D. Sun) and jinbaoqin@163.com (J. Qin).

${ }^{1}$ These authors contributed equally to this work. 
Figure S-1. The FTIR spectrum of Gelatin and GelMA

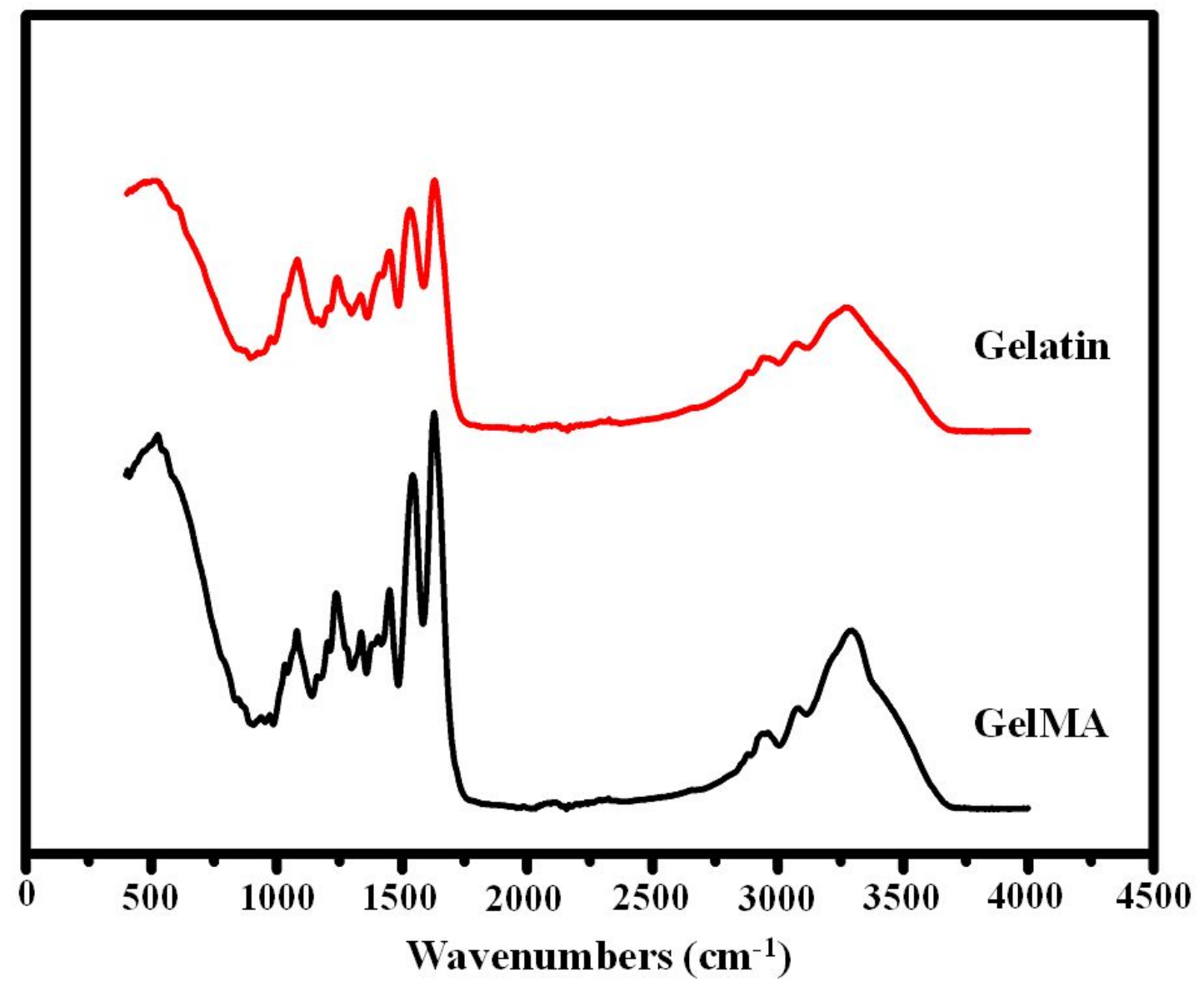

Figure S-2. The optical image of GelMA/Ca ${ }^{2+}$-Alginate hydrogel before and after UV irradiation

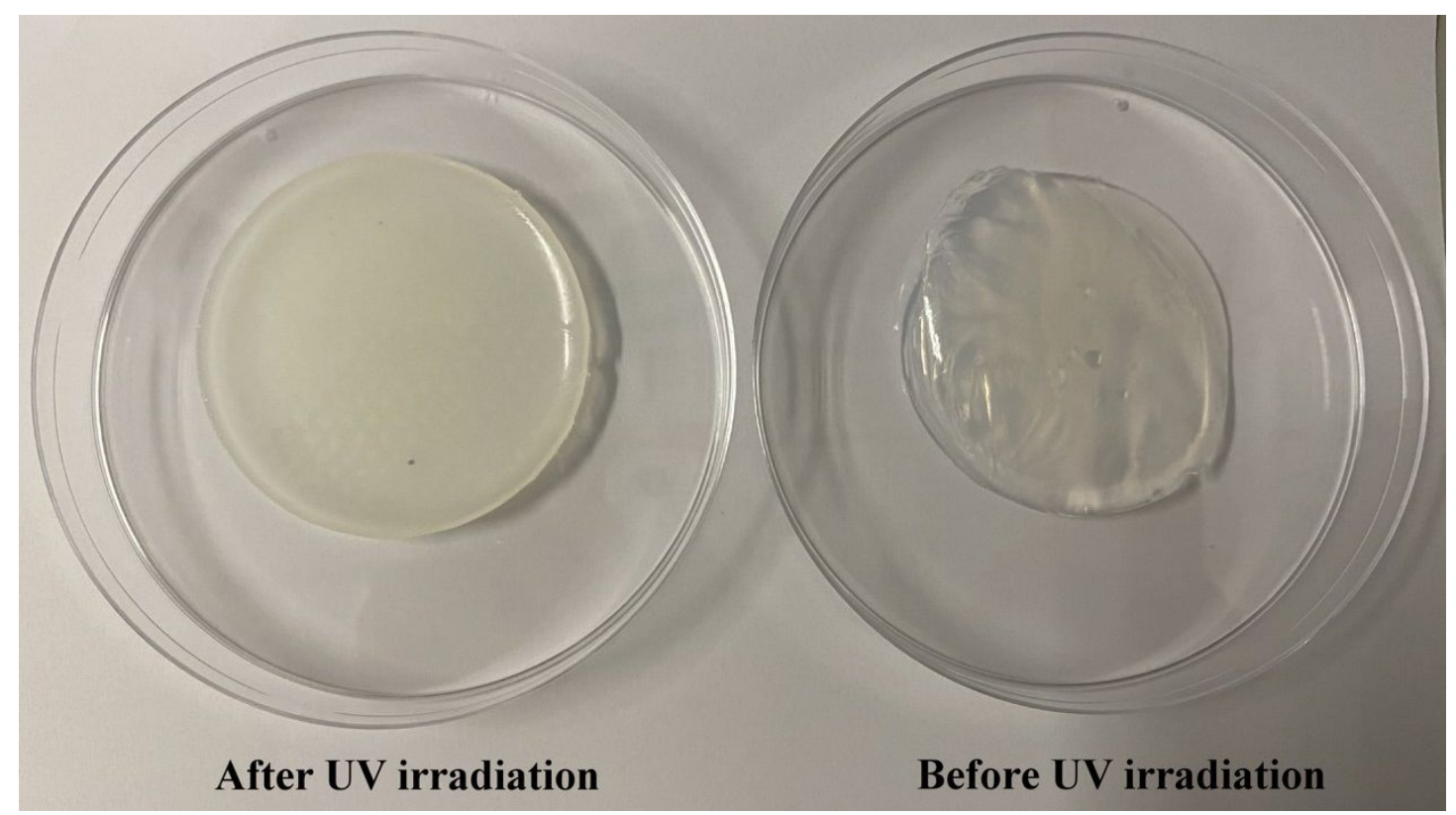


Figure S-3. Cytotoxicity and cell viability assay

a) The cytotoxicity of GMT/DN hydrogel scaffolds. b) The effect of graphene on proliferation of RSC96 cells.

a

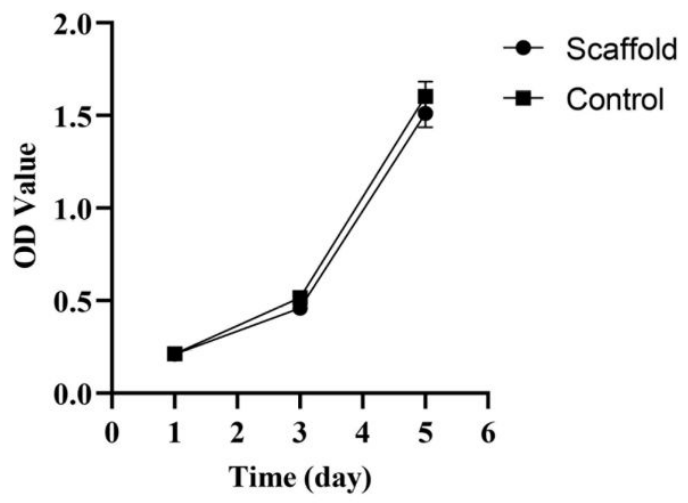

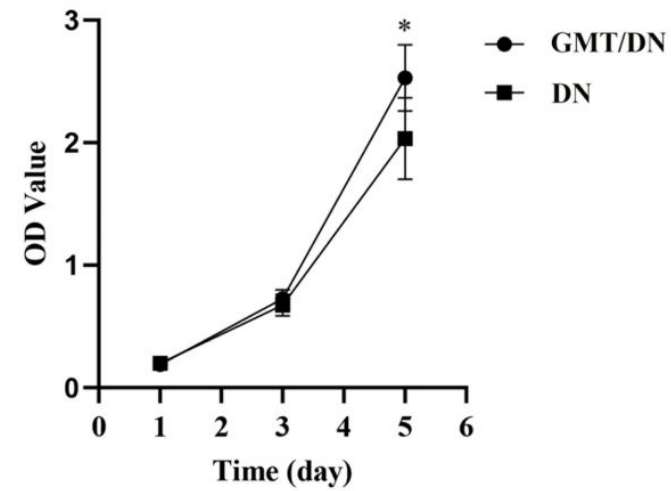

Figure S-4. Morphology of Schwann cells seeded on the surface of GMT/DN hydrogel scaffolds

a) Schwann cells seeded on the surface of GMT/DN hydrogel scaffolds, the black dashed lines represent the location of graphene mesh. b) Control group, Schwann cells seeded on the surface of pure DN hydrogel scaffolds.
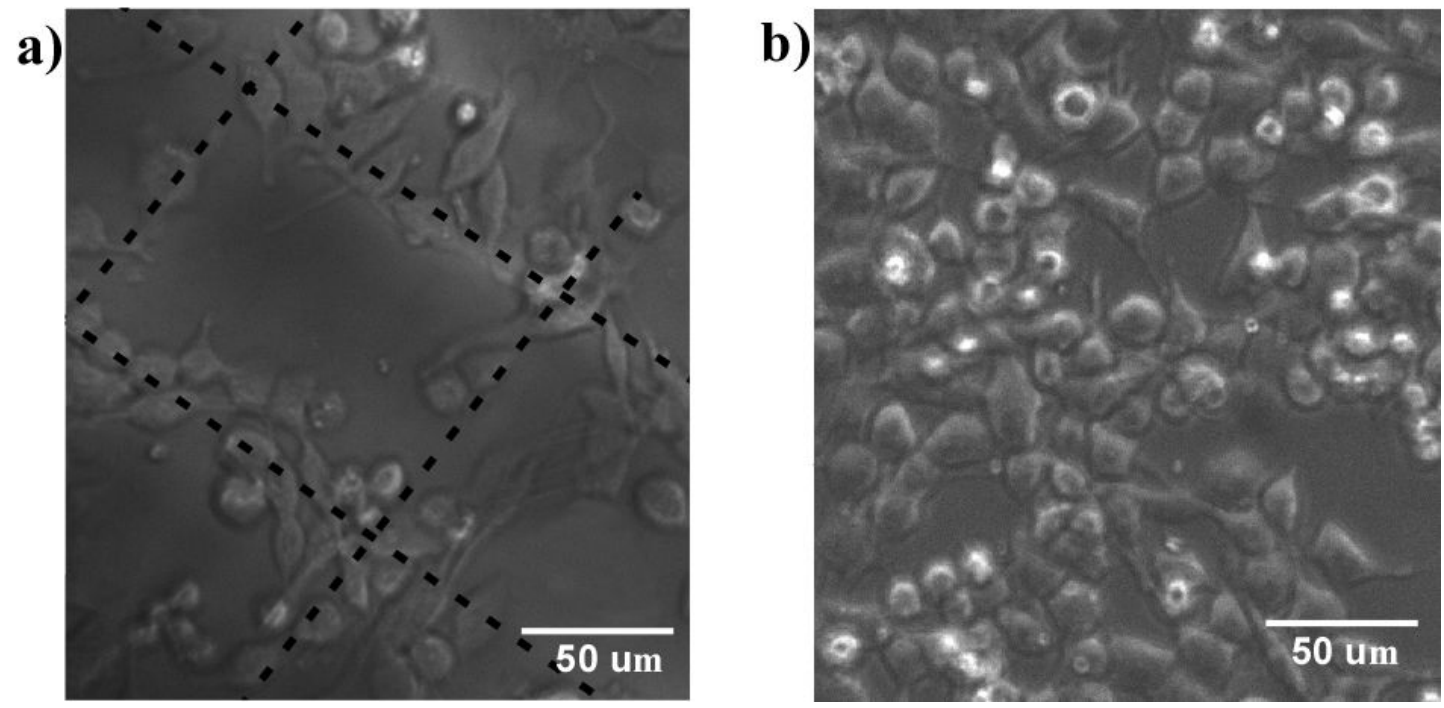

Figure S-5. Photographs of nerve regeneration scaffold immediately after implantation

a) Exposed sciatic nerve. b) Cutting $1 \mathrm{~cm}$ sciatic nerve. c) GMT/DN hydrogel nerve 
scaffold implantation.

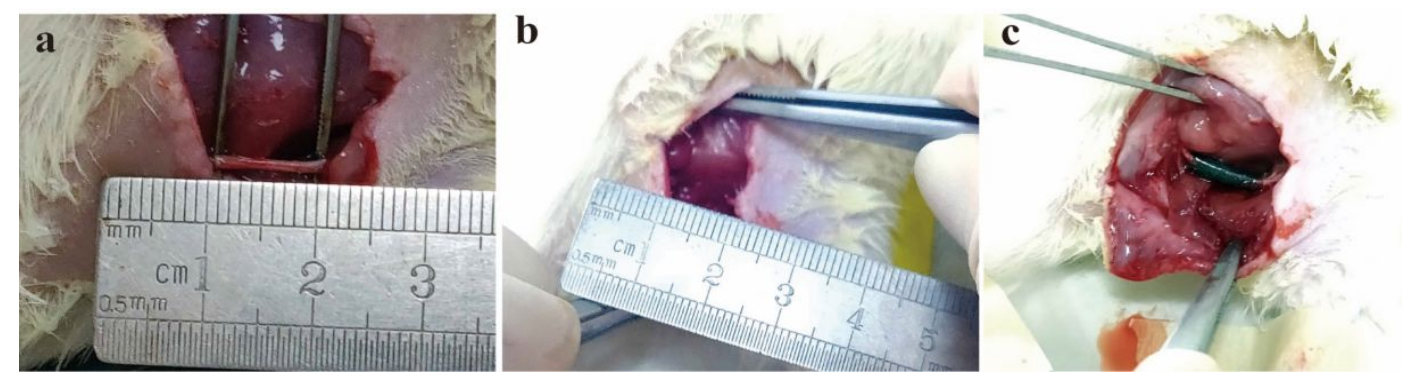

Figure S-6. The regenerative capacity of peripheral nerve in groups of autologous graft, scaffold, and scaffold+Netrin-1 a) Immunofluorescence staining of vWF in perineural suture. Scale bar: $50 \mu \mathrm{m}$. b) Microvessel density by vWF staining in perineural suture. c) Immunofluorescence staining of $\mathrm{vWF}$ in intraneural structure. Scale bar: $100 \mu \mathrm{m}$. d) Microvessel density by vWF staining in intraneural structure. e) Immunofluorescence staining of Sox10 of sciatic nerves. Scale bar: $100 \mu \mathrm{m}$. f) Analysis of Sox 10 positive cells of sciatic nerves. 


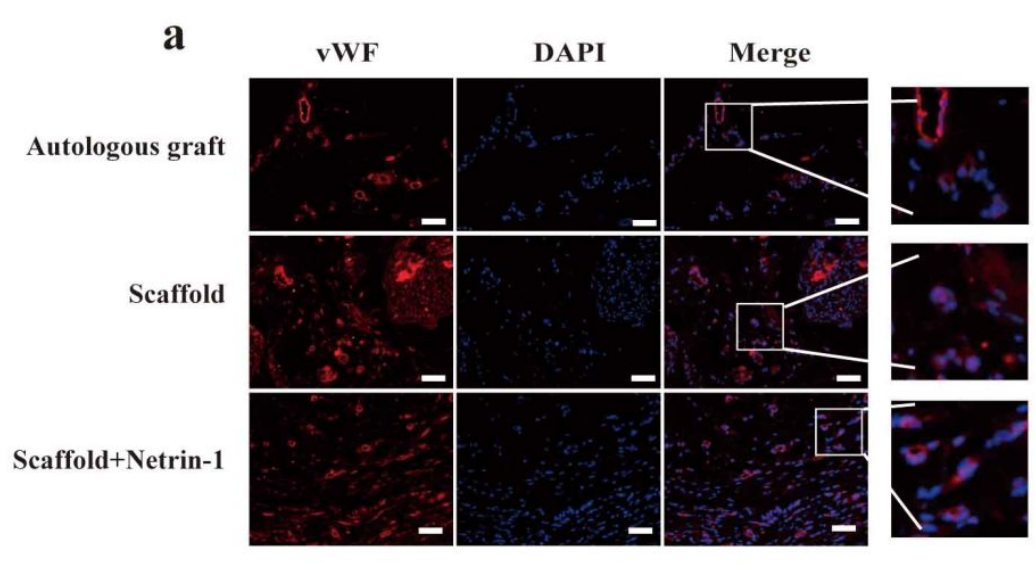

b

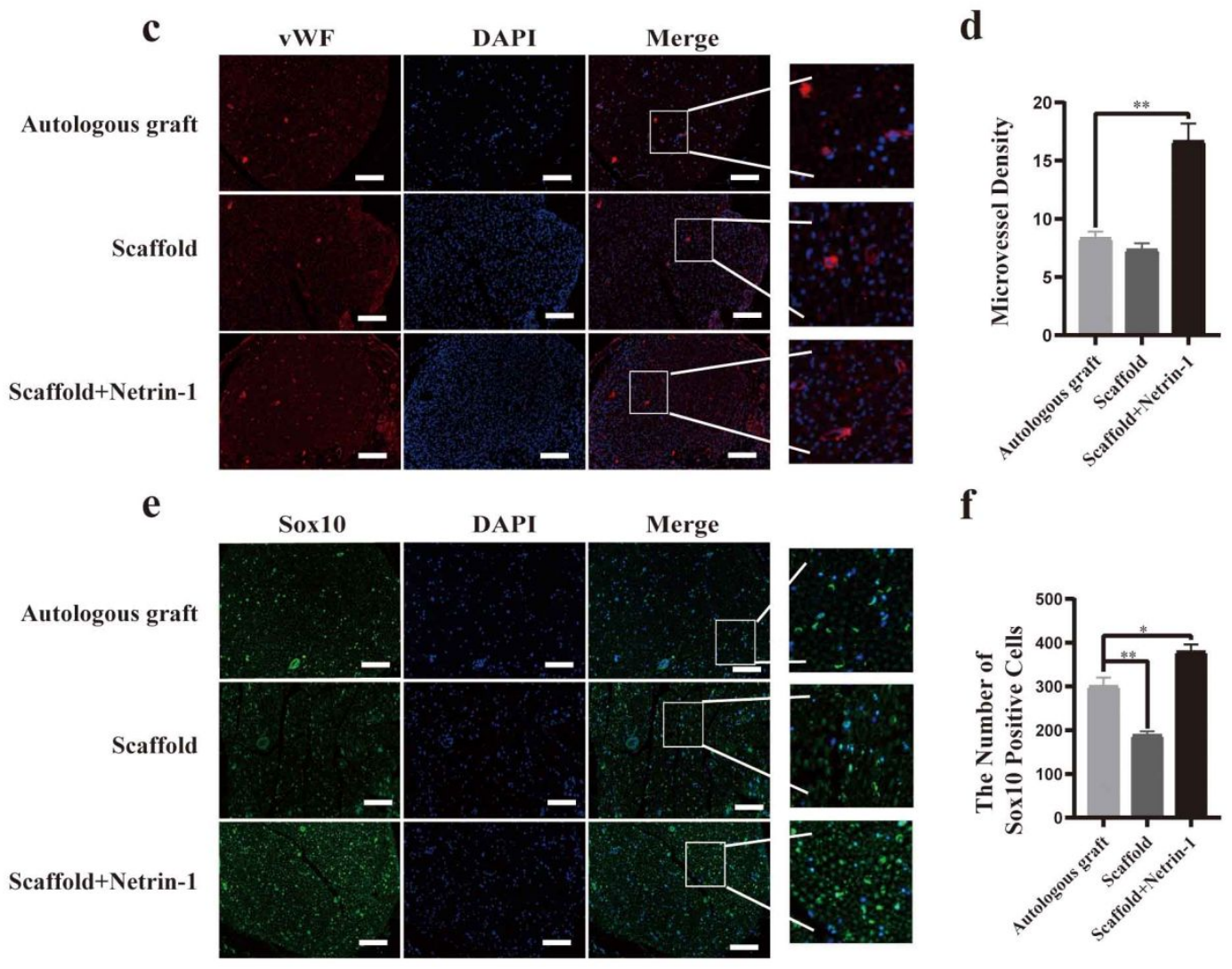

Table S-1. The comparison of graphene based peripheral nerve regeneration conduit

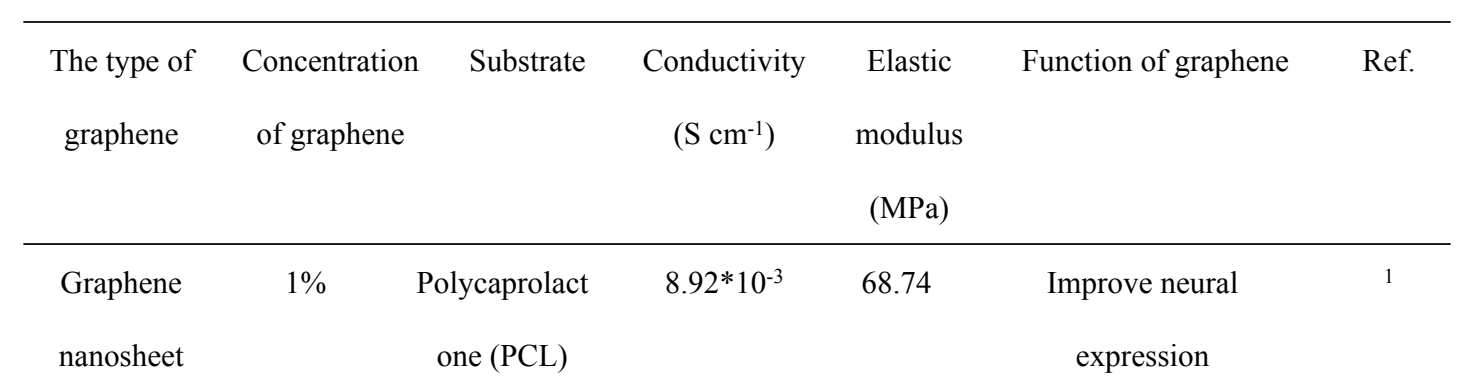




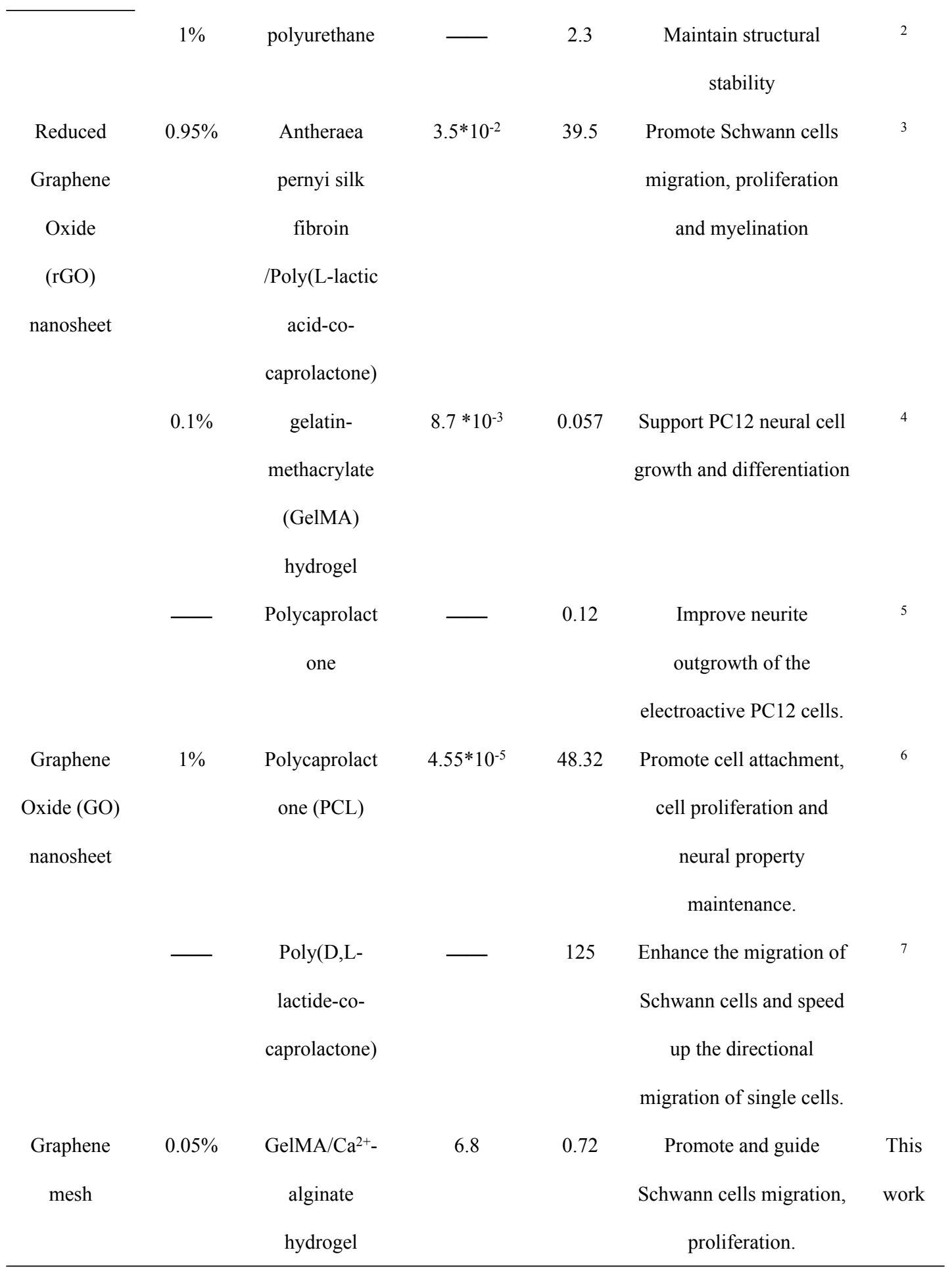

\section{References :}

1. Qian, Y.; Zhao, X.; Han, Q.; Chen, W.; Li, H.; Yuan, W. An Integrated Multi-Layer 3D-Fabrication of PDA/RGD Coated Graphene Loaded PCL Nanoscaffold for Peripheral Nerve Restoration. Nat. Commun. 2018, 9 , 1-16. 
2. Lee, T.-H.; Yen, C.-T.; Hsu, S.-h. Preparation of Polyurethane-Graphene Nanocomposite and Evaluation of Neurovascular Regeneration. ACS Biomater. Sci. Eng. 2019, 6, 597-609.

3. Wang, J.; Cheng, Y.; Chen, L.; Zhu, T.; Ye, K.; Jia, C.; Wang, H.; Zhu, M.; Fan, C.; Mo, X. In Vitro and In Vivo Studies of Electroactive Reduced Graphene Oxide-Modified Nanofiber Scaffolds for Peripheral Nerve Regeneration. Acta Biomater. 2019, 84, 98-113.

4. Park, J.; Jeon, J.; Kim, B.; Lee, M. S.; Park, S.; Lim, J.; Yi, J.; Lee, H.; Yang, H. S.; Lee, J. Y. Electrically Conductive Hydrogel Nerve Guidance Conduits for Peripheral Nerve Regeneration. Adv. Funct. Mater. 2020, 30 , 2003759.

5. Zhang, Z.; Jørgensen, M. L.; Wang, Z.; Amagat, J.; Wang, Y.; Li, Q.; Dong, M.; Chen, M. 3D Anisotropic Photocatalytic Architectures As Bioactive Nerve Guidance Conduits for Peripheral Neural Regeneration. Biomaterials 2020, 253, 120108.

6. Qian, Y.; Song, J.; Zhao, X.; Chen, W.; Ouyang, Y.; Yuan, W.; Fan, C. 3D Fabrication with Integration Molding of a Graphene Oxide/Polycaprolactone Nanoscaffold for Neurite Regeneration and Angiogenesis. Adv. Sci. 2018, 5, 1700499. 7. Zhang, D.; Yao, Y.; Duan, Y.; Yu, X.; Shi, H.; Nakkala, J. R.; Zuo, X.; Hong, L.; Mao, Z.; Gao, C. Surface-Anchored Graphene Oxide Nanosheets on CellScale Micropatterned Poly (d, 1-lactide-co-caprolactone) Conduits Promote Peripheral Nerve Regeneration. ACS Appl. Mater. Interfaces 2020, 12, 7915-7930. 